\title{
ON A DISTANCE INVARIANT UNDER MÖBIUS TRANSFORMATIONS IN $\mathbf{R}^{n}$
}

\author{
HEINZ LEUTWILER
}

\section{Introduction}

The distance referred to in the title is the following: Let $\Omega$ be a domain in $\mathbf{R}^{n}$ and set

$$
\varrho_{\Omega}(x, y)=\log \frac{\sup \left\{\frac{h(x)}{h(y)}: h>0, \text { harmonic on } \Omega\right\}}{\inf \left\{\frac{h(x)}{h(y)}: h>0, \text { harmonic on } \Omega\right\}}
$$

for all $x, y \in \Omega$. Then $\varrho_{\Omega}$ defines a (complete) distance on $\Omega$, provided the positive harmonic functions separate the points of $\Omega$.

In case $\Omega=B_{n}$, the unit ball in $\mathbf{R}^{n}, \varrho_{\Omega}$ is - up to a factor $n$ - equal to the distance function $\delta_{B_{n}}$, defined by the Poincaré metric $d s=2\left(1-\|x\|^{2}\right)^{-1}\|d x\|$ (Theorem 4.2).

The distance $\varrho_{\Omega}$ has the following invariance property: For any Möbius transformation $\gamma$, we have $\varrho_{\gamma \Omega}(\gamma x, \gamma y)=\varrho_{\Omega}(x, y)$ for all $x, y \in \Omega$, where $\gamma \Omega$ denotes the image of $\Omega$ under $\gamma$ (see Theorem 4.1). The invariance of $\varrho_{\Omega}$ is based on a generalization of Kelvin's transformation to arbitrary Möbius transformations $\gamma$ (Lemma 2.1).

In Section 3 we establish sharp bounds for the positive harmonic functions in $B_{n}$ and the upper half-space $H_{n}$. These will be used in Sections 4 and 5, in the latter to find an explicit expression for the so-called Harnack distance $d_{B_{n}}$ of $B_{n}$, a distance generally introduced by J. Köhn in [4].

The generalization of Definition 1.1 given in Section 6 incorporates other invariant distances such as the classical Cayley-Klein distance or the well-known Carathéodory distance. 


\section{Transformation-preserving harmonicity}

In $\mathbf{R}^{n}$ all similarities preserve harmonicity. So, according to Kelvin's theorem, does the transformation $T: h \rightarrow \tilde{h}$, where

$$
\tilde{h}(x)=\frac{1}{\|x\|^{n-2}} h\left(\frac{x}{\|x\|^{2}}\right) .
$$

The following lemma is based on the observation that, with these two special cases, any Möbius transformation in $\mathbf{R}^{n}$ gives rise to a transformation of this sort.

Recall that the full Möbius group $\hat{M}\left(\mathbf{R}^{n}\right)$ is the group generated by all similarities together with the inversion

$$
J: x \rightarrow x^{*}=\frac{x}{\|x\|^{2}} .
$$

As in Ahlfors [1], for any $\gamma \in \hat{M}\left(\mathbf{R}^{n}\right)$, we denote by $\left|\gamma^{\prime}(x)\right|$ the unique positive real number such that

$$
\frac{\gamma^{\prime}(x)}{\left|\gamma^{\prime}(x)\right|} \in O(n)
$$

Here $O(n)$ denotes the orthogonal group and $\gamma^{\prime}(x)$ the Jacobian matrix of $\gamma$ at the point $x$. Observing now that $\left|J^{\prime}(x)\right|=\|x\|^{-2}$, (2.1) reads as follows:

$$
\tilde{h}(x)=\left|J^{\prime}(x)\right|^{(n-2) / 2} h(J x) .
$$

Hence, if $\gamma$ denotes either a similarity or the inversion $J$, we have the following result: The transformation

$$
T_{\gamma}: h \rightarrow \tilde{h} \text {, where } \tilde{h}(x)=\left|\gamma^{\prime}(x)\right|^{(n-2) / 2} h(\gamma x),
$$

preserves harmonicity. Moreover, if $\gamma_{1}$ and $\gamma_{2}$ are any two Möbius transformations with this property, an application of the chain rule shows that the composition $\gamma_{1} \circ \gamma_{2}$ again has this property.

We therefore conclude that

Lemma 2.1. For any Möbius transformation $\gamma \in \hat{M}\left(\mathbf{R}^{n}\right)$ the transformation $T_{\gamma}$ defined by 2.4 preserves harmonicity and positivity.

Of particular interest is the Möbius transformation $\gamma_{a}$ defined for $a=0$ by the identity mapping and for each $a \in \mathbf{R}^{n}$ with $0<\|a\|<1$ by

$$
\gamma_{a}(x)=\frac{\left(1-\|a\|^{2}\right)(x-a)-\|x-a\|^{2} a}{1-2 a x+\|a\|^{2}\|x\|^{2}}
$$

where $a x:=a_{1} x_{1}+\ldots+a_{n} x_{n}$ denotes the usual scalar product (see Ahlfors [1]). The transformation $\gamma_{a}$ maps the unit ball $B_{n}$ onto itself, thereby sending the point $a$ 
into zero. Actually the most general $\gamma \in \hat{M}\left(\mathbf{R}^{n}\right)$ satisfying these conditions is of the form $k \gamma_{a}$, where $k \in O(n)$, as shown in [1].

According to [1] (Chapter II, formula 30), we have

$$
\left|\gamma_{a}^{\prime}(x)\right|=\frac{1-\|a\|^{2}}{1-2 a x+\|a\|^{2}\|x\|^{2}}=\frac{1-\|a\|^{2}}{\|a\|^{2}\left\|x-a^{*}\right\|^{2}},
$$

where $a^{*}=a /\|a\|^{2}(a \neq 0)$.

An application of Lemma 2.1 therefore yields the following result, which can also be verified directly:

Corollary 2.2. Let $\gamma_{a}, 0<\|a\|<1$, denote the Möbius transformation defined by (2.5). Then the transformation $T_{a}: h \rightarrow \tilde{h}$, where

$$
\tilde{h}(x)=\frac{1}{\left\|x-a^{*}\right\|^{n-2}} h\left(\gamma_{a} x\right) \quad\left(x \in B_{n}\right),
$$

maps one positive harmonic function on the unit ball $B_{n}$ into another.

Remark. In order to have a better insight into Lemma 2.1, we introduce for any $\gamma \in \hat{M}\left(\mathbf{R}^{n}\right)$ and any $C^{2}$-function $u$ on $\Omega \subset \mathbf{R}^{n}$ the notion $u_{\gamma}$, defined by

$$
u_{\gamma}(x)=\left|\gamma^{\prime}(x)\right|^{(n-2) / 2} u(\gamma x) \quad\left(x \in \gamma^{-1} \Omega\right) .
$$

Denoting the Laplace operator $\left(\partial^{2} / \partial x_{1}^{2}\right)+\ldots+\left(\partial^{2} / \partial x_{n}^{2}\right)$ by $\Delta$ as usual, we claim that for any $C^{2}$-function $u$ on $\Omega$ we have

$$
\Delta\left(u_{\gamma}\right)=\left|\gamma^{\prime}\right|^{2}(\Delta u)_{\gamma} .
$$

The proof again consists in showing that if formula (2.9) holds for $\gamma_{1}, \gamma_{2} \in \hat{M}\left(\mathbf{R}^{n}\right)$ (for all $u \in C^{2}(\Omega)$ ), it also holds for the composition $\gamma_{1} \circ \gamma_{2}$. We there use the fact that $u_{\gamma_{1} \circ \gamma_{2}}=\left(u_{\gamma_{1}}\right)_{\gamma_{2}}$.

\section{Sharp bounds for positive harmonic functions in $B_{n}$ and $H_{n}$}

We shall now use Lemma 2.1 in order to find sharp bounds for the quotients $h(x) / h(y)$ if $h$ ranges over all positive harmonic functions, defined on the unit ball $B_{n}$, or the upper half space $H_{n}:=\left\{\left(x_{1}, \ldots, x_{n}\right): x_{n}>0\right\}$, respectively. In order to avoid duplication we shall, however, set it up in such a way that it also serves the purpose of the next section.

Let $\Omega$ be a domain in $\mathbf{R}^{n}(n \geqq 2)$ and set for $x, y \in \Omega$

$$
D_{\Omega}(x, y)=\log \sup \left\{\frac{h(x)}{h(y)}: h>0, \text { harmonic on } \Omega\right\} .
$$


Then we have

$$
e^{-D_{\Omega}(y, x)} \leqq \frac{h(x)}{h(y)} \leqq e^{D_{\Omega}(x, y)}
$$

for all positive harmonic functions $h$ on $\Omega$ and all $x, y \in \Omega$. Furthermore, both bounds are attained.

Lemma 3.1. Let $\gamma$ be a Möbius transformation, mapping a domain $\Omega \subset \mathbf{R}^{n}$ onto a domain $\widetilde{\Omega}$. Then we have

$$
D_{\Omega}(x, y)=D_{\tilde{\Omega}}(\gamma x, \gamma y)+\frac{n-2}{2} \log \frac{\left|\gamma^{\prime}(x)\right|}{\left|\gamma^{\prime}(y)\right|}
$$

for all $x, y \in \Omega$.

Proof. Let $u>0$ be harmonic on $\widetilde{\Omega}$ and set

$$
h(z)=\left|\gamma^{\prime}(z)\right|^{(n-2) / 2} u(\gamma z) \quad(z \in \Omega) .
$$

Then, on account of Lemma $2.1, h$ is harmonic on $\Omega$.

From

we conclude that

$$
\frac{u(\gamma x)}{u(\gamma y)} \leqq e^{D_{\Omega}(\gamma x, \gamma y)}
$$

$$
\frac{h(x)}{h(y)} \leqq e^{D_{\Omega}(\gamma x, \gamma y)}\left(\frac{\left|\gamma^{\prime}(x)\right|}{\left|\gamma^{\prime}(y)\right|}\right)^{(n-2) / 2} .
$$

Since the upper bound on the right is actually attained, Lemma 3.1 follows.

Choosing $\Omega=B_{n}$, the unit ball in $\mathbf{R}^{n}$, the classical Harnack inequality

$$
h(x) \leqq \frac{1+\|x\|}{(1-\|x\|)^{n-1}} h(0) \quad\left(x \in B_{n}\right),
$$

valid for all $h>0$, harmonic on $B_{n}$, results in

$$
D(x, 0)=D_{B_{n}}(x, 0)=\log \frac{1+\|x\|}{(1-\|x\|)^{n-1}} .
$$

In order to determine $D$ for any pair of points $(x, y) \in B_{n} \times B_{n}$, we apply the Möbius transformation $\gamma_{y}$ defined in (2.5) (with $a$ replaced by $y$ ). According to Lemma 3.1 we then have

$$
\begin{aligned}
& D(x, y)=D\left(\gamma_{y} x, 0\right)+\frac{n-2}{2} \log \frac{\left|\gamma_{y}^{\prime}(x)\right|}{\left|\gamma_{y}^{\prime}(y)\right|} \\
& =\log \frac{1+\left\|\gamma_{y} x\right\|}{\left(1-\left\|\gamma_{y} x\right\|\right)^{n-1}}+\frac{n-2}{2} \log \frac{\left|\gamma_{y}^{\prime}(x)\right|}{\left|\gamma_{y}^{\prime}(y)\right|} .
\end{aligned}
$$

But $\left\|\gamma_{y} x\right\|=\|x-y\| /[x, y]$, where $[x, y]^{2}=1-2 x y+\|x\|^{2}\|y\|^{2}$ (see [1], Chapter II, 
formula 32). Inserting this and the expression (2.6) for $\left|\gamma_{y}^{\prime}(x)\right|$ we find that

$$
D(x, y)=\log \frac{([x, y]+\|x-y\|)\left(1-\|y\|^{2}\right)^{n-2}}{([x, y]-\|x-y\|)^{n-1}} .
$$

Using the identity $\left(1-\|x\|^{2}\right)\left(1-\|y\|^{2}\right)=[x, y]^{2}-\|x-y\|^{2}$, we get the more convenient expression

$$
D_{B_{n}}(x, y)=\frac{n}{2} \log \frac{[x, y]+\|x-y\|}{[x, y]-\|x-y\|}+\frac{n-2}{2} \log \frac{1-\|y\|^{2}}{1-\|x\|^{2}} .
$$

Summarizing we have

Theorem 3.2. Every positive harmonic function $h$ on the unit ball $B_{n} \quad(n \geqq 2)$ satisfies the sharp inequality

$$
\begin{gathered}
\left(\frac{\sqrt{1-2 x y+\|x\|^{2}\|y\|^{2}}-\|x-y\|}{\sqrt{1-2 x y+\|x\|^{2}\|y\|^{2}}+\|x-y\|}\right)^{n / 2}\left(\frac{1-\|y\|^{2}}{1-\|x\|^{2}}\right)^{(n-2) / 2} \\
\leqq \frac{h(x)}{h(y)} \leqq\left(\frac{\sqrt{1-2 x y+\|x\|^{2}\|y\|^{2}}+\|x-y\|}{\sqrt{1-2 x y+\|x\|^{2}\|y\|^{2}}-\|x-y\|}\right)^{n / 2}\left(\frac{1-\|y\|^{2}}{1-\|x\|^{2}}\right)^{(n-2) / 2}
\end{gathered}
$$

for arbitrary $x, y \in B_{n}$.

We emphasize that in this double inequality both sides are attained.

Introducing the distance function $\delta_{B_{n}}$ with respect to the Poincaré metric

$$
d s=\frac{2\|d x\|}{1-\|x\|^{2}} \quad\left(x \in B_{n}\right),
$$

i.e., the function

$$
\delta(x, y)=\delta_{B_{n}}(x, y)=\log \frac{\sqrt{1-2 x y+\|x\|^{2}\|y\|^{2}}+\|x-y\|}{\sqrt{1-2 x y+\|x\|^{2}\|y\|^{2}}-\|x-y\|} \quad\left(x, y \in B_{n}\right),
$$

the inequality in Theorem 3.2 can also be written as follows:

$$
e^{-(n / 2) \delta(x, y)}\left(\frac{1-\|y\|^{2}}{1-\|x\|^{2}}\right)^{(n-2) / 2} \leqq \frac{h(x)}{h(y)} \leqq e^{(n / 2) \delta(x, y)}\left(\frac{1-\|y\|^{2}}{1-\|x\|^{2}}\right)^{(n-2) / 2} .
$$

This form is especially convenient if one wants to transfer the estimate to the upper half-space $H_{n}$.

To this end we consider the Möbius transformation $\gamma$ defined by

$$
\gamma(x)=\frac{1}{\left\|x-e_{n}\right\|^{2}}\left(2 x_{1}, \ldots, 2 x_{n-1}, 1-\|x\|^{2}\right), \quad x=\left(x_{1}, \ldots, x_{n}\right),
$$

where $e_{n}$ denotes the $n^{\text {th }}$ coordinate vector. It maps $B_{n}$ bijectively onto $H_{n}$. Introducing the distance $\delta_{H_{n}}$ defined in $H_{n}$ by the Poincaré metric

$$
d s=\frac{\|d z\|}{z_{n}}, \quad z=\left(z_{1}, \ldots, z_{n}\right) \in H_{n},
$$


i.e., the function

$$
\delta_{H_{n}}(u, v)=\log \frac{\|u-\bar{v}\|+\|u-v\|}{\|u-\bar{v}\|-\|u-v\|},
$$

where $u, v \in H_{n}, u=\left(u_{1}, \ldots, u_{n}\right), v=\left(v_{1}, \ldots, v_{n}\right)$ and $\bar{v}=\left(v_{1}, \ldots, v_{n-1},-v_{n}\right)$, we know that

$$
\delta_{B_{n}}(x, y)=\delta_{H_{n}}(\gamma x, \gamma y)
$$

for all $x, y \in B_{n}$.

The application of Lemma 3.1 now yields

$$
D_{B_{n}}(x, y)=D_{H_{n}}(\gamma x, \gamma y)+\frac{n-2}{2} \log \frac{\left|\gamma^{\prime}(x)\right|}{\left|\gamma^{\prime}(y)\right|} .
$$

Since $\left|\gamma^{\prime}(x)\right|=2\left\|x-e_{n}\right\|^{-2}$, we conclude from (3.6) and (3.8) that

$$
D_{H_{n}}(\gamma x, \gamma y)=\frac{n}{2} \delta_{B_{n}}(x, y)+\frac{n-2}{2} \log \frac{\left(1-\|y\|^{2}\right)\left\|x-e_{n}\right\|^{2}}{\left\|y-e_{n}\right\|^{2}\left(1-\|x\|^{2}\right)} .
$$

Hence, setting $u=\gamma x, v=\gamma y$, we infer from (3.10) and (3.13) that

So we have proved

$$
D_{H_{n}}(u, v)=\frac{n}{2} \delta_{H_{n}}(u, v)+\frac{n-2}{2} \log \frac{v_{n}}{u_{n}} .
$$

Theorem 3.3. Every positive harmonic function $h$ on the upper half-space $H_{n}$ $(n \geqq 2)$ satisfies the sharp inequality

$$
\left(\frac{\|x-\bar{y}\|-\|x-y\|}{\|x-\bar{y}\|+\|x-y\|}\right)^{n / 2}\left(\frac{y_{n}}{x_{n}}\right)^{(n-2) / 2} \leqq \frac{h(x)}{h(y)} \leqq\left(\frac{\|x-\bar{y}\|+\|x-y\|}{\|x-\bar{y}\|-\|x-y\|}\right)^{n / 2}\left(\frac{y_{n}}{x_{n}}\right)^{(n-2) / 2},
$$

for arbitrary $x=\left(x_{1}, \ldots, x_{n}\right), y=\left(y_{1}, \ldots, y_{n}\right) \in H_{n}$.

\section{The invariant distance $\varrho_{\Omega}$}

The function $D_{\Omega}(x, y)$, defined under (3.1) for an arbitrary domain $\Omega \subset \mathbf{R}^{n}$ $(n \geqq 2)$, is nonnegative and satisfies the triangle inequality: For all $x, y, z \in \Omega$ we have $D_{\Omega}(x, z) \leqq D_{\Omega}(x, y)+D_{\Omega}(y, z)$. However, it is not necessarily symmetric. We therefore consider the sum

$$
\varrho_{\Omega}(x, y)=D_{\Omega}(x, y)+D_{\Omega}(y, x)
$$

for $x, y \in \Omega$. Then $\varrho_{\Omega}$ defines a pseudo-distance, which of course can also be introduced by (1.1).

It is a distance if and only if the positive harmonic functions on $\Omega$ separate the points. If the latter is the case, then in fact $\varrho_{\Omega}$ defines a complete distance, which always induces the euclidean topology on $\Omega$ (see the remark in Section 5). 
The main feature of this seemingly new distance is, as already mentioned in the introduction, the following invariance property:

Theorem 4.1. For any Möbius transformation $\gamma$ mapping a domain $\Omega \subset \mathbf{R}^{n}$ onto a domain $\widetilde{\Omega}$ we have

$$
\varrho_{\Omega}(x, y)=\varrho_{\Omega}(y x, \gamma y)
$$

for all $x, y \in \Omega$.

Proof. Immediate consequence of Lemma 3.1 and Definition (4.1).

From (3.6) and (3.8) we conclude

Theorem 4.2. In the unit ball $B_{n}(n \geqq 2)$ we have

$$
\varrho_{B_{n}}=n \delta_{B_{n}},
$$

where $\delta_{B_{n}}$ denotes the Poincaré distance (3.8).

Analogously we infer from (3.14):

Theorem 4.3. In the upper half-space $H_{n}(n \geqq 2)$ we have

$$
\varrho_{H_{n}}=n \delta_{H_{n}},
$$

where $\delta_{H_{n}}$ denotes the Poincaré distance (3.12).

In $\mathbf{R}^{1}$ the harmonic functions are the affine-linear ones. Defining the Poincaré distance on the open interval $(-1,1)$ as in $(3.7)$ by the differential $2\left(1-x^{2}\right)^{-1}|d x|$, Theorem 4.2 remains true for the case $n=1$. Indeed, considered on the open interval $I=(a, b)$, the positive affine-linear functions define the distance function

$$
\varrho_{I}(x, y)=\left|\log \frac{\frac{x-a}{x-b}}{\frac{y-a}{y-b}}\right|=|\log (x, y, a, b)|,
$$

where $(x, y, a, b)$ denotes the cross-ratio of the four points $x, y, a, b$. This is not surprising, since in the case of the unit disc in $\mathbf{R}^{2}$, the Poincaré distance can also be defined as a cross-ratio:

$$
\delta_{B_{2}}(x, y)=|\log (x, y, \xi, \eta)| .
$$

Here $x, y \in B_{2}$ and $\xi$ and $\eta$ denote the points on the unit circle $C$ lying also on the circle orthogonal to $C$, and passing through the points $x$ and $y$ (see e.g. [1], Chapter I, and Chapter II for an extension to $B_{n}$ ).

It is an obvious but important fact that for any two domains $\Omega_{1}$ and $\Omega_{2} \subset \mathbf{R}^{n}$ $(n \geqq 2)$ we have

$$
\Omega_{1} \subset \Omega_{2} \Rightarrow \varrho_{\Omega_{1}}(x, y) \geqq \varrho_{\Omega_{2}}(x, y), \text { for all } x, y \in \Omega_{1} .
$$


Consequently, we find for example that for any mapping $f$ which maps $B_{n}$ onto a subdomain $\Omega$ of $B_{n}$ and satisfies the condition

$$
\varrho_{\Omega}(f(x), f(y)) \leqq \varrho_{B_{n}}(x, y)\left(=n \delta_{B_{n}}(x, y)\right)
$$

for all $x, y \in B_{n}$, we have

$$
\delta_{B_{n}}(f(x), f(y)) \leqq \delta_{B_{n}}(x, y) \quad\left(x, y \in B_{n}\right) .
$$

If, in addition, $f(0)=0$, it follows from the monotonicity of the function $r \rightarrow \delta_{B_{n}}(r, 0)$ that

$$
\|f(x)\| \leqq\|x\|,
$$

for all $x \in B_{n}$.

Assuming further that the function $f$ which maps $B_{n}$ onto the subdomain $\Omega$ of $B_{n}$ and satisfies (4.6) is differentiable, we infer from (4.7) that for all $x \in B_{n}$

$$
\left\|f^{\prime}(x)\right\| \leqq \frac{1-\|f(x)\|^{2}}{1-\|x\|^{2}} .
$$

Here, as usual, we define the norm of the Jacobian matrix $A=f^{\prime}(x)$ by $\|A\|=$ $=\sup \left\{\|A v\|: v \in \mathbf{R}^{n},\|v\|=1\right\}$.

In case $n=2$, the condition (4.6) is automatically satisfied, provided $f$ is holomorphic. Indeed, given any nonconstant holomorphic function $f: B_{2} \rightarrow B_{2}$, we know that its image $\Omega=f\left(B_{2}\right)$ is open. Furthermore, for every positive harmonic function $h$ on $\Omega$ the composition $h \circ f$ is positive and harmonic on $B_{2}$, so that (4.6) follows immediately from Definition (4.1). The inequalities (4.7)-(4.9) therefore imply Schwarz's lemma.

In $\mathbf{R}^{n}$ ( $n \geqq 3$ ), (4.6) is satisfied, according to Theorem 4.1, at least if $f$ denotes a Möbius transformation which maps $B_{n}$ into $B_{n}$.

\section{The Harnack distance}

Another possibility to "symmetrize" the function $D_{\Omega}(x, y)$, defined in Section 3 for an arbitrary domain $\Omega \subset \mathbf{R}^{n}(n \geqq 2)$, is to set

$$
d_{\Omega}(x, y)=\max \left[D_{\Omega}(x, y), D_{\Omega}(y, x)\right]
$$

for $x, y \in \Omega$. Then $d_{\Omega}$ defines a pseudo distance already introduced by J. Köhn [4] under the name Harnack (pseudo-) distance for $\Omega$. Indeed, for all $x, y \in \Omega$, we have

$$
\begin{aligned}
d_{\Omega}(x, y) & =\log \inf \left\{a \mid \frac{1}{a} \leqq \frac{h(x)}{h(y)} \leqq a: \forall h \in \mathscr{H}^{+}(\Omega)\right\} \\
& =\sup \left\{|\log h(x)-\log h(y)|: h \in \mathscr{H}^{+}(\Omega)\right\},
\end{aligned}
$$

where $\mathscr{H}^{+}(\Omega)$ denotes the set of positive harmonic functions on $\Omega$. 
From the inequality $\max (a, b) \leqq a+b \leqq 2 \max (a, b)$, valid for all positive real numbers $a, b$, we immediately deduce

Lemma 5.1. On every domain $\Omega$ in $\mathbf{R}^{n}$, the pseudo-distances $\varrho_{\Omega}$ and $d_{\Omega}$ are equivalent. More precisely, we have

$$
d_{\Omega} \leqq \varrho_{\Omega} \leqq 2 d_{\Omega} .
$$

Remark. In [4] (Korollar 3) it has been shown that in case the positive harmonic functions separate the points of $\Omega, d_{\Omega}$ defines a complete metric which induces the usual topology on $\Omega$. Consequently, by Lemma 5.1, the same is true for the distance $\varrho_{\Omega}$. Combining (5.3) with (4.2) yields

Corollary 5.2. Let $\gamma$ be a Möbius transformation, mapping a domain $\Omega \subset \mathbf{R}^{n}$ onto a domain $\widetilde{\Omega}$. Then

for all $x, y \in \Omega$.

$$
\frac{1}{2} d_{\Omega}(x, y) \leqq d_{\Omega}(\gamma x, \gamma y) \leqq 2 d_{\Omega}(x, y)
$$

On the other hand, it follows from Lemma 5.1 and Theorem 4.2 (and Theorem 4.3) that in $B_{n}$ (and $H_{n}$ ) the Harnack distance $d_{B_{n}}$ (and $d_{H_{n}}$ ) is equivalent to the Poincaré distance $\delta_{B_{n}}$ (and $\delta_{H_{n}}$ ). More precisely, we have

$$
\frac{n}{2} \delta_{B_{n}} \leqq d_{B_{n}} \leqq n \delta_{B_{n}}, \quad \text { respectively } \quad \frac{n}{2} \delta_{H_{n}} \leqq d_{H_{n}} \leqq n \delta_{H_{n}} .
$$

On account of (3.6) and (3.14) we can in fact explicitly compute $d_{B_{n}}$ and $d_{H_{n}}$.

Theorem 5.3. The Harnack distance $d_{B_{n}}$ of the unit ball $B_{n}(n \geqq 2)$ is given by

$$
d_{B_{n}}(x, y)=\frac{n}{2} \delta_{B_{n}}(x, y)+\frac{n-2}{2}\left|\log \frac{1-\|x\|^{2}}{1-\|y\|^{2}}\right|
$$

for $x, y \in B_{n}$, whereas the Harnack distance $d_{H_{n}}$ of the upper half space $H_{n}(n \geqq 2)$ is given by

$$
d_{H_{n}}(x, y)=\frac{n}{2} \delta_{H_{n}}(x, y)+\frac{n-2}{2}\left|\log \frac{x_{n}}{y_{n}}\right|,
$$

where $x=\left(x_{1}, \ldots, x_{n}\right), y=\left(y_{1}, \ldots, y_{n}\right) \in H_{n}$.

For general domains $\Omega \subset \mathbf{R}^{n}$ with a boundary $\partial \Omega \neq \emptyset$ we can easily estimate the Harnack distance from below in terms of the euclidean distance dist $(z, \partial \Omega)$ from the point $z \in \Omega$ to the boundary $\partial \Omega$ :

Lemma 5.4. If $\Omega$ is a proper subdomain of $\mathbf{R}^{n} \quad(n \geqq 3)$, then

$$
d_{\Omega}(x, y) \geqq(n-2)\left|\log \frac{\operatorname{dist}(x, \partial \Omega)}{\operatorname{dist}(y, \partial \Omega)}\right|
$$

for all $x, y \in \Omega$. 
Proof. Let $x, y \in \Omega$, where w.l.o.g. dist $(y, \partial \Omega) \leqq \operatorname{dist}(x, \partial \Omega)$. Choose a point $p \in \partial \Omega$ such that $\|y-p\|=\operatorname{dist}(y, \partial \Omega)$. Then, by definition, we also have dist $(x, \partial \Omega) \leqq$ $\leqq\|x-p\|$.

Consider next the Newtonian kernel $k(z)=\|z-p\|^{2-n}\left(z \in \mathbf{R}^{n} \backslash\{p\}\right)$. Since $k$ is positive and harmonic on $\Omega$, we conclude from (5.2) that

$$
\begin{gathered}
d_{\Omega}(x, y) \geqq \log \|y-p\|^{2-n}-\log \|x-p\|^{2-n} \\
\geqq(2-n) \log \operatorname{dist}(y, \partial \Omega)+(n-2) \log \operatorname{dist}(x, \partial \Omega),
\end{gathered}
$$

and (5.7) hence follows.

From (5.3) and (5.7) we then get

Corollary 5.5. If $\Omega$ is a proper subdomain of $\mathbf{R}^{n}(n \geqq 3)$, then

for all $x, y \in \Omega$.

$$
\varrho_{\Omega}(x, y) \geqq(n-2)\left|\log \frac{\operatorname{dist}(x, \partial \Omega)}{\operatorname{dist}(y, \partial \Omega)}\right|
$$

We finally mention that equality holds in (5.7) and (5.8) in case $\Omega=\mathbf{R}^{n} \backslash\{0\}$. Thus both estimates are sharp.

\section{A general definition of $\varrho$}

In this last section we are going to extend Definition (1.1) of the distance $\varrho_{\Omega}$ in two ways. On the one hand, it is important to consider additional distances defined by subsets of the set $\mathscr{H}^{+}(\Omega)$ of all positive harmonic functions on $\Omega \subset \mathbf{R}^{n}$, on the other hand it becomes necessary to incorporate, besides the Laplace equation, further partial differential equations.

The most general setting in which our distance makes sense is the following one. Let $\Omega$ be an abstract set and $\mathscr{F}$ an arbitrary class of (strictly) positive, real-valued functions, defined on $\Omega$, with the property that the following Harnack-type inequality holds: For any pair of points $x, y \in \Omega$ there is a constant $a=a(x, y)>0$ such that

$$
h(x) \leqq a h(y)
$$

for all $h \in \mathscr{F}$.

Definition. For all $x, y \in \Omega$ we set

$$
\varrho_{\Omega, \mathscr{F}}(x, y)=\log \frac{\sup \left\{\frac{h(x)}{h(y)}: h \in \mathscr{F}\right\}}{\inf \left\{\frac{h(x)}{h(y)}: h \in \mathscr{F}\right\}} .
$$

Then $\varrho_{\Omega, \mathscr{F}}$ defines a pseudo-distance, which is a distance if and only if the family $\mathscr{F}$ separates the points of $\Omega$ (linearly if $1 \notin \mathscr{F}$ ). 
Parallel to $\varrho_{\Omega, \mathscr{F}}$ we also introduce the Harnack pseudo-distance

$$
d_{\Omega, \mathscr{F}}(x, y)=\log \inf \left\{a: \frac{1}{a} \leqq \frac{h(x)}{h(y)} \leqq a, \quad \forall h \in \mathscr{F}\right\},
$$

already introduced in this generality by $\mathbf{J} . \mathrm{Köhn}$ in [4]. It is clear that again $\varrho_{\Omega, \mathscr{F}}$ and $d_{\Omega, \mathscr{F}}$ are equivalent pseudo-distances. Also, many properties proved in [4] for $d_{\Omega, \mathscr{F}}$ remain true for $\varrho_{\Omega, \mathscr{F}}$. Observing for example that for any two families $\mathscr{F}_{\mathbf{1}}$ and $\mathscr{F}_{2}$ of positive functions on $\Omega$, satisfying the condition (6.1), we have

$$
\mathscr{F}_{1} \subset \mathscr{F}_{2} \Rightarrow \varrho_{\Omega, \mathscr{F}_{1}} \leqq \varrho_{\Omega}, \mathscr{F}_{2},
$$

we immediately conclude that the following generalization of the "principle of hyperbolic length" must hold:

Lemma 6.1. Let $\mathscr{F}(\tilde{\mathscr{F}})$ denote a class of positive functions, defined on some abstract set $\Omega(\tilde{\Omega})$. Assume further that $\mathscr{F}$ and $\tilde{\mathscr{F}}$ satisfy (6.1). Then, for any mapping $F: \Omega \rightarrow \widetilde{\Omega}$ with the property that for each $h \in \tilde{\mathscr{F}}, h \circ F$ is an element of $\mathscr{F}$, we have

$$
\varrho_{\tilde{\Omega}, \tilde{\mathscr{F}}}[F(x), F(y)] \leqq \varrho_{\Omega, \mathscr{F}}(x, y)
$$

for all $x, y \in \Omega$.

Our first choice for a subset of the classical harmonic functions is the family of all positive, affine functions on a convex domain $\Omega \subset \mathbf{R}^{n}$. Here we have basically the same situation as in the one-dimensional case treated in Section 4.

Theorem 6.2. Let $\Omega$ be a convex open subset of $\mathbf{R}^{n}$, containing no straight line, and let $\mathscr{F}$ be the set of positive affine functions on $\Omega$. Then we have

$$
\varrho_{\Omega, F}(x, y)=|\log (x, y, a, b)|,
$$

where $(x, y, a, b)$ denotes the cross-ratio of $x, y \in \Omega$ and the points $a, b$, in which the straight line through $x$ and $y$ intersects the boundary $\partial \Omega$.

Clearly, in case the straight line through $x$ and $y$ has only one intersection point, say $a$, we set $b=\infty$.

Proof. Let $g$ denote the straight line passing through the points $x, y \in \Omega$. On account of what has been said in Section 4 about the one-dimensional case it clearly suffices to show that every non-constant, affine function $l$ on $g$, which is positive on $g \cap \Omega$, is the restriction of an affine function on $\mathbf{R}^{n}$, which is positive on $\Omega$. To this end we consider the supporting hyperplane $E$ for the convex set $\Omega$ in that intersection point $a$ or $b \in \partial \Omega$, in which $l$ assumes the smaller value. The unique affine function on $\mathbf{R}^{n}$ which extends $l$ and is constant on $E$ then has the desired properties.

For the case where $\Omega \subset \mathbf{R}^{2}$ is defined by a conic, the distance (6.6) has already been introduced by Cayley in 1859. However, it was F. Klein (see his collected 
works) who noticed in 1871 that if $\Omega$ denotes the unit disc, the distance (6.6) agrees - up to a constant factor - with the non-euclidean metric used in his (non-conformal) model of hyperbolic geometry. Therefore, transforming this distance with the aid of the mapping $\zeta=z /\left(1+\sqrt{1-|z|^{2}}\right)$ from $B_{2}$ onto itself, one again gets - up to a factor 2 - the distance (3.8) (for $n=2$ ).

Note also that the distance (6.6) is obviously invariant under projectivities.

Remark. Theorem 6.2 indicates that for any convex lineless subset $K$ of a linear space $V$ one might also look, besides the so-called part metric (see e.g. H. S. Bear [2] for its definition), at the following distance function. Let $x, y$ be two points of the same part of $K$. We say that the segment $[x, y]$ extends in $K$ by the pair of positive numbers $(\alpha, \beta)$ if and only if $x+\alpha(x-y)$ and $y+\beta(y-x)$ are elements of $K$. Then

$$
\varrho_{K}(x, y)=\inf \left\{\log \left(1+\frac{1}{\alpha}\right)+\log \left(1+\frac{1}{\beta}\right):[x, y] \text { extends in } K \text { by }(\alpha, \beta)\right\}
$$

defines a distance on the parts of $K$ which is equivalent to the part metric $d_{K}$. In fact, we have $d_{K} \leqq \varrho_{K} \leqq 2 d_{K}$, similar to (5.3).

In $\mathbf{C}^{n}$, identified with $\mathbf{R}^{2 n}$, every domain $\Omega$ has its invariant distance $\varrho_{\Omega}$, defined according to (1.1) by the set $\mathscr{H}^{+}(\Omega)$ of all positive harmonic functions on $\Omega$. This distance, however, is not invariant under the class of holomorphic mappings on $\Omega$. In order to define a distance of this sort, we must consider a subclass $\mathscr{F}$ of $\mathscr{H}^{+}(\Omega)$ which is invariant under such mappings. Hence a natural choice for $\mathscr{F}$ in this situation is the following one:

$$
\mathscr{F}=\{\operatorname{Re} f \mid f: \Omega \rightarrow \mathbf{C} \text { holomorphic, } \operatorname{Re} f>0\} .
$$

This set $\mathscr{F}$ is actually meaningful on any domain $\Omega$ of a complex manifold $M$ of dimension $n$. We therefore formulate the following proposition in this generality.

Proposition 6.3. Let $\Omega$ be a domain in a complex manifold $M$ of dimension $n$ and let $\mathscr{F}$ be defined as in (6.8). Then we have

$$
\varrho_{\Omega, F}=2 d_{\Omega, \mathscr{F}}=2 c_{\Omega},
$$

where $c_{\Omega}$ denotes the classical Carathéodory (pseudo-) distance, defined by

$$
c_{\Omega}(x, y)=\sup \left\{\delta_{B_{2}}(f(x), f(y)) \mid f: \Omega \rightarrow \mathbf{C} \text { holomorphic, }|f|<1\right\}
$$

for all $x, y \in \Omega$.

Proof. Since $\varrho \leqq 2 d$ (analogously to the proof of (5.3)), it suffices to show that $\varrho \geqq 2 c$ and $d \leqq c$.

In order to prove that $\varrho \geqq 2 c$ we take a function $f$ holomorphic on $\Omega$ with $|f|<1$ and apply Lemma 6.1 with $\mathscr{F}=\{\operatorname{Re} g \mid g$ holomorphic on $\Omega, \operatorname{Re} g>0\}$, 
$\widetilde{\Omega}=B_{2}=\{z \in \mathbf{C}:|z|<1\}, \tilde{\mathscr{F}}=\mathscr{H}^{+}\left(B_{2}\right)$ and $F=f$. There results

$$
\varrho_{B_{2}}(f(x), f(y)) \leqq \varrho(x, y), \text { for all } x, y \in \Omega .
$$

By Theorem 4.2, $\varrho_{B_{2}}=2 \delta_{B_{2}}$, and hence we have

$$
2 \delta_{B_{2}}(f(x), f(y)) \leqq \varrho(x, y)
$$

for all holomorphic functions $f$ on $\Omega$ with $|f|<1$. Consequently, by (6.10), we must have $2 c_{\Omega}(x, y) \leqq \varrho(x, y)$, for all $x, y \in \Omega$.

For the proof of the remaining inequality $d \leqq c$, we first note that for all $x, y \in H_{n}$ we have

$$
\delta_{H_{n}}(x, y) \geqq\left|\log \frac{x_{n}}{y_{n}}\right|,
$$

as we easily conclude from (3.12). In case $n=2$, this yields the following estimate for the Poincare distance $\delta_{\tilde{H}_{2}}$ of the right half plane $\widetilde{H}_{2}=\{w \in C: \operatorname{Re} w>0\}$ :

for all $w_{1}, w_{2} \in \tilde{H}_{2}$.

$$
\delta_{\tilde{H}_{2}}\left(w_{1}, w_{2}\right) \geqq\left|\log \frac{\operatorname{Re} w_{1}}{\operatorname{Re} w_{2}}\right|
$$

Let now $g$ be holomorphic on $\Omega$ with $\operatorname{Re} g>0$ and denote by $\varphi$ the two-dimensional Möbius transformation $w \rightarrow(w-1)(w+1)^{-1}$, which maps the right half-plane $\tilde{H}_{2}$ onto the unit disc $B_{2}$. Setting $f=\varphi \circ g$ yields a holomorphic function $f$ with $|f|<1$. Hence, according to Definition (6.10) of $c=c_{\Omega}$, we have

$$
c(x, y) \geqq \delta_{B_{2}}(f(x), f(y))=\delta_{\tilde{H}_{2}}(g(x), g(y)) \geqq\left|\log \frac{\operatorname{Re} g(x)}{\operatorname{Re} g(y)}\right| .
$$

Setting $h=\operatorname{Re} g$ we conclude that $e^{-c(x, y)} \leqq h(x) h(y)^{-1} \leqq e^{c(x, y)}$ and hence $d(x, y) \leqq$ $c(x, y)$ for all $x, y \in \Omega$.

Another reasonable choice for a set $\mathscr{F}$, provided $\Omega$ is again a domain on a complex manifold $M$ of dimension $n$, is the following one:

$$
\mathscr{F}^{\prime}=\{h \mid h>0 \text {, pluriharmonic on } \Omega\} .
$$

By (6.4) the corresponding pseudo-distance $\varrho_{\Omega, \mathscr{F}}$, dominates $\varrho_{\Omega, \mathscr{F}}$, defined with respect to the set (6.8). On the other hand, $\varrho_{\Omega}, \mathscr{F}$, itself is dominated by a multiple of the so-called Kobayashi pseudo-distance $k_{\Omega}$ (see e.g. [3], p. 45, for its definition). Indeed, on account of Lemma 6.1, every holomorphic mapping $f: B_{2} \rightarrow \Omega$ satisfies the estimate

$$
\varrho_{\Omega, \mathscr{F}^{\prime}}(f(x), f(y)) \leqq \varrho_{B_{2}}(x, y), \text { for } x, y \in B_{2} .
$$

The inequality $\varrho_{\Omega, \mathscr{T}} \leqq 4 k_{\Omega}$ therefore follows from the maximality property (Proposition 1.4 of Chapter IV in [3]) of $k_{\Omega}$.

A further class of harmonic functions that one might consider is the set of multiply harmonic functions. Here we immediately conclude for example from the one-dimensional (complex) case: 
Proposition 6.4. Let $\varrho$ denote the distance defined on the polydisc $\left\{\left(z_{1}, \ldots, z_{n}\right) \in \mathbf{C}^{n}:\left|z_{i}\right|<1, i=1, \ldots, n\right\}$ by the positive multiply harmonic functions and let $d$ be the corresponding Harnack distance. Then we have

$$
\varrho(z, \zeta)=2 d(z, \zeta)=2\left[\delta\left(z_{1}, \zeta_{1}\right)+\ldots+\delta\left(z_{n}, \zeta_{n}\right)\right],
$$

where $z=\left(z_{1}, \ldots, z_{n}\right), \zeta=\left(\zeta_{1}, \ldots, \zeta_{n}\right)$ and $\delta=\delta_{B_{2}}$.

Turning finally to differential equations other than the Laplace equation, we only recall that the Harnack inequality (6.1) holds for instance for the positive $C^{2}$-solutions of any linear elliptic partial differential equation of second order, provided the coefficients are locally Hölder-continuous. More generally, if $(\Omega, \mathscr{H})$ denotes any harmonic space in the sense of M. Brelot, the positive harmonic functions $\mathscr{H}^{+}(\Omega)$ satisfy the Harnack inequality.

Remark. It is important to note that in case $(\Omega, \mathscr{H})$ denotes a harmonic space in the sense of M. Brelot and $\varphi$ a (strictly) positive continuous function on $\Omega$, the distance $\varrho_{\Omega, \mathscr{H}}$ defined with respect to $\mathscr{H}^{+}(\Omega)$ does not change if one replaces the sheaf $\mathscr{H}$ by the quotient sheaf $\mathscr{H}_{\varphi}=\{h / \varphi: h \in \mathscr{H}\}$. This sheds some light on Theorem 4.1.

It is known that on any Riemannian manifold the $C^{2}$-solutions of the corresponding Laplace-Beltrami equation define a harmonic space in the sense of M. Brelot. An interesting example of such an equation is the so-called Weinstein equation,

$$
\Delta u+\frac{k}{x_{n}} \frac{\partial u}{\partial x_{n}}=0 \quad(k \in \mathbf{R}),
$$

defined on the upper half-space $H_{n}(n \geqq 3)$. It includes the classical harmonic functions $(k=0)$, as well as the hyperbolic ones $(k=2-n)$. The proof of the following result will appear in [6].

Proposition 6.5. Let @ denote the distance defined on $H_{n}(n \geqq 3)$ by the positive $C^{2}$-solutions of the Weinstein equation (6.12). Then we have

$$
\varrho=(n-1+|k-1|) \delta_{H_{n}},
$$

where $\delta_{H_{n}}$ denotes the Poincaré distance (3.12).

We conclude with the following two remarks:

1) There are interesting connections between $\varrho_{\Omega}$ and the class $\operatorname{BMO}(\Omega)$ of all harmonic functions of bounded mean oscillation, defined on the domain $\Omega \subset \mathbf{R}^{n}$. This topic will be dealt with in [5].

2) Besides the distance $\varrho_{\Omega}\left(\Omega\right.$ a domain in $\left.\mathbf{R}^{n}\right)$, it will also be worthwhile to study the differential metric $\sigma_{\Omega}$, defined on $\Omega \times \mathbf{R}^{n}$ by

$$
\sigma_{\Omega}(x, v)=\sup \left\{\frac{d h(x) v}{h(x)}: h \in \mathscr{H}^{+}(\Omega)\right\}-\inf \left\{\frac{d h(x) v}{h(x)}: h \in \mathscr{H}^{+}(\Omega)\right\},
$$


where $d h(x)$ denotes the differential of the harmonic function $h$ at the point $x \in \Omega$. It is easy to see that $\sigma_{\Omega}$ gives rise to a pseudo-distance, which is also invariant under Möbius transformations.

In case $\Omega=B_{n}, \sigma_{\Omega}$ is equal to $n$ times the Poincaré differential metric (3.7). It would be interesting to characterize all the domains $\Omega$ in $\mathbf{R}^{n}$ whose differential metric is Riemannian. We also do not know on what domains $\Omega$ the distance $\varrho_{\Omega}$ is the "integrated form" of $\sigma_{\Omega}$.

\section{References}

[1] Ahlfors, L. V.: Möbius transformations in several dimensions. - Ordway Lectures in Mathematics, University of Minnesota, 1981.

[2] BEAR, H. S.: Lectures on Gleason parts. - Lecture Notes in Mathematics 121. Springer-Verlag, Berlin-Heidelberg-New York, 1970.

[3] KobayaShi, S.: Hyperbolic manifolds and holomorphic mappings. - Marcel Dekker, Inc., New York, 1970.

[4] KöHN, J.: Die Harnacksche Metrik in der Theorie der harmonischen Funktionen. - Math. Z. 91, 1966, 50-64.

[5] LEUTWILER, H.: On BMO and the torsion function.

[6] Leutwiler, H.: Best constants in the Harnack inequality for the Weinstein equation. - Aequationes Math.

Universität Erlangen-Nürnberg

Mathematisches Institut

Bismarckstraße 1 1/2

D-8520 Erlangen

Federal Republic of Germany

Received 28 November 1983

(Approved 25 February, 1987) 